\title{
Update für multidisziplinäres Wissen
}

D er Ärzteverband Deutscher Allergologen (ÄDA), die Deutsche Gesellschaft für Allergologie und klinische Immunologie (DGAKI) sowie die Gesellschaft für Pädiatrische Allergologie und Umweltmedizin (GPA) laden Sie herzlich ein zum 6. Deutschen Allergiekongress. In diesem Jahr übernimmt der ÄDA die Schirmherrschaft über die Veranstaltung. In der Kur- und Kongressstadt Wiesbaden erwartet Sie ein umfangreiches und hochaktuelles Tagungsangebot: Darin verbinden wir ein Update auf dem Gebiet der allergologischen Forschung mit praxisorientierter Fortbildung. Die Breite des Programmangebots spiegelt die Tatsache wider, dass die Allergologie traditionell ein multidisziplinäres Fach ist.

In den vergangenen Jahren hat sich das Klima für Allergiepatienten und ihre Ärzte erheblich verschlechtert. Gesundheitspolitik und Selbstverwaltung ignorieren die Tatsache, dass nicht nur die Zahl der Allergiker stetig zunimmt, sondern auch die Schwere ihrer Erkrankungen. Für alle in der Allergologie Tätigen gilt es, dieser Entwicklung entschieden entgegenzutreten, um eine ausreichende medizinische Versorgung von Allergiekranken sicherzustellen. Dies ist nicht nur im Sinne der Patienten, son-

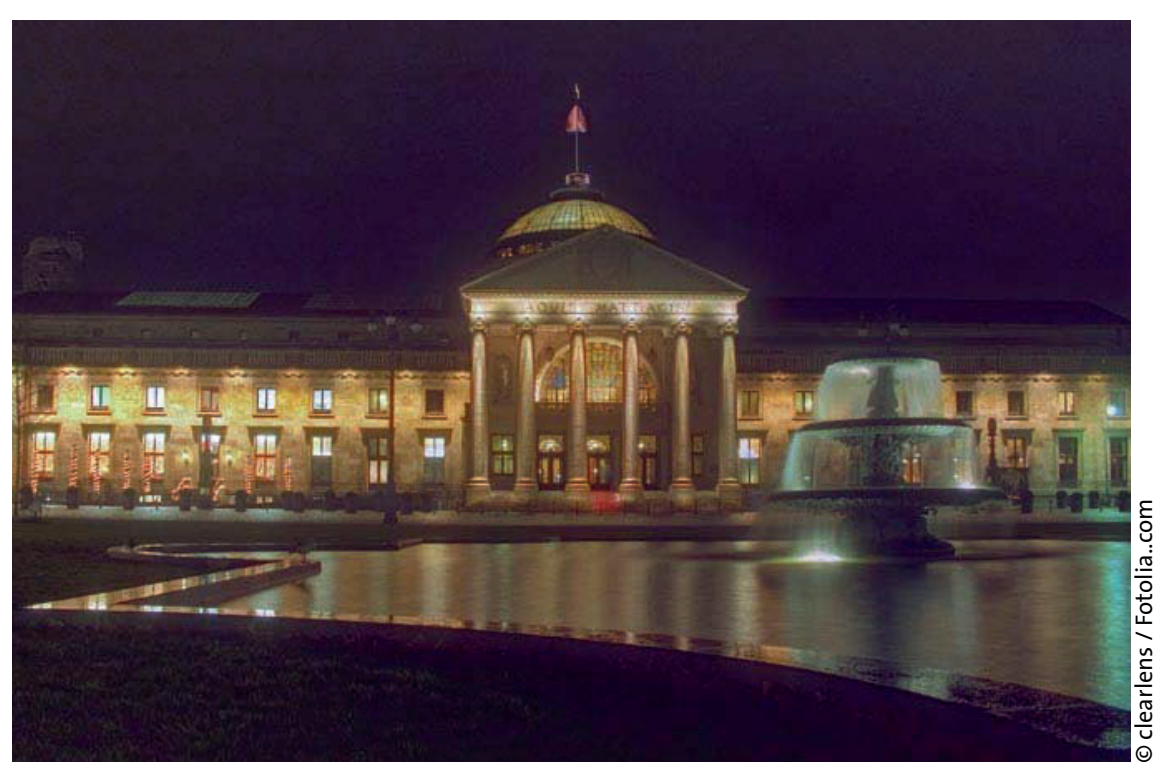

Das Wiesbadener Kurhaus inmitten eines Parkensembles mit Wasserspielen umrahmt den Kongress bei Tag und Nacht glanzvoll.

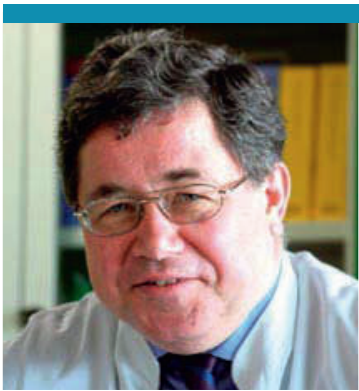

Prof. Dr. Hans F. Merk, Hautklinik der Medizinischen Fakultät, Universitätsklinikum der RWTH Aachen, Präsident der ÄDA

dern auch im Sinne der ökonomischen Verwendung der finanziellen Ressourcen der gesamten Volkswirtschaft.

Der Schulterschluss der drei großen deutschen allergologischen Fachgesellschaften bei der Kongressgestaltung seit dem 1. Gemeinsamen Deutschen Allergiekongress in Aachen 2004 leistet einen wichtiger Beitrag zur Verwirklichung der genannten Ziele.

Tagungspräsidenten des diesjährigen Kongresses sind Prof. Dr. Ludger Klimek und Prof. Dr. Wolfgang Schlenter. Beide organisieren bereits zum zweiten Mal einen nationalen Allergiekongress in Wiesbaden. Professor Klimek ist zudem

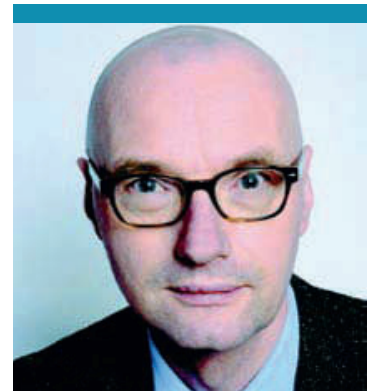

Prof. Dr. Harald Renz, Institut für Laboratoriumsmedizin, Universitätsklinikum Gießen und Marburg, Präsident der DGAKI

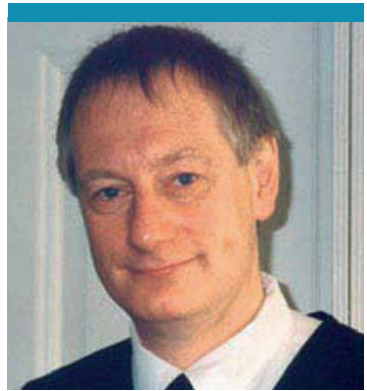

Prof. Dr. Albrecht Bufe, Abteilung für experimentelle Pneumologie, Universitätsklinik Bergmannsheil, Bochum, Präsident der GPA verantwortlich für die beliebten Fortbildungen, die jedes Frühjahr im Kloster Eberbach stattfinden. Somit stehen die Tagungspräsidenten also für einen informativen, wissenschaftlich anspruchsvollen und spannenden Kongress auf höchstem Niveau, bei dem auch der persönliche Austausch unter den Teilnehmern nicht zu kurz kommen wird. Wir danken den beiden und allen an der Vorbereitung dieser Tagung beteiligten Kolleginnen und Kollegen der drei Fachgesellschaften sowie der neu zum Team gestoßenen Agentur wikonect.

In Wiesbaden wird übrigens nicht nur über Gesundheit referiert und diskutiert. Tun Sie es Dostojewski, Brahms oder Jawlensky gleich und testen Sie die belebende Wirkung der unter- wie überirdischen Schätze der Stadt am eigenen Leib: Das legendäre Mineral- und Thermalwasser sprudelt im Quellenviertel auf Schritt und Tritt aus großen Tiefen an die Oberfläche. Trinken Sie reichlich davon und bewundern Sie beim irischrömischen Bad die Kurarchitektur. So können Sie sich während der Kongresstage gleichzeitig vom Alltag erholen und kehren erfrischt in die Universität, Klinik oder Praxis zurück.

Wir freuen uns darauf, Sie bald in Wiesbaden begrüßen zu können, einem Veranstaltungsort der zwischen Frankfurt und dem Rheingau viel Flair und kulturelle Angebote bereithält.

Prof. Dr. Hans F. Merk Prof. Dr. Harald Renz

Prof. Dr. Albrecht Bufe 